\title{
Appendix to: ABA and The Combinatorics of Morphological Features
}

\author{
Jonathan David Bobaljik* \\ University of Connecticut $\&$ \\ Leibniz-Zentrum Allgemeine \\ Sprachwissenschaft (ZAS) \\ Storrs, CT, USA \& Berlin, Germany
}

\author{
Uli Sauerland ${ }^{\dagger \ddagger}$ \\ Leibniz-Zentrum Allgemeine \\ Sprachwissenschaft (ZAS) \\ Berlin, Germany
}

Final authors' version, October 2017

\section{A Appendix: Beyond three cells}

As we move beyond a three-cell space, the system grows and changes in various ways. Unlike the two- and three-cell spaces, we will not walk through all examples in as much detail, but will call attention to various points and provide some more general discussion in the abstract. Our presentation in this appendix is abbreviated compared to that in the main text and we hope to return to this in the future. For now, our main purpose is to show that feature combinatorics alone becomes more restrictive as the number of cells increases.

A three-cell paradigm space allows for $2^{3}-1=7$ features, and thus $2^{7}=128$ logically possible inventories. We showed in the previous section that Validity reduces that set to 96 and Validity plus Minimality further reduces the number of contenders to 3 feature inventories. By countenancing arbitrary ordering of the rules of exponence (sequences), each of the three minimally valid inventories generates 3 of the $B_{3}=5$ partitions of a 3-member set: none derives the undifferentiated partition. Adding Pānini as a restriction reduces that further: from the three minimal valid feature inventories, only one partition can be generated-the maximally differentiated partition.

The four-cell case allows $B_{4}=15$ partitions (paradigms) including the maximally differentiated partition. Logically, $2^{B_{4}-1}=16384$ different valid partition sets exist. If there were no constraints on feature inventories or rules of exponence, this is the number of possible states of the world that we could

* jonathan.bobaljik@uconn.edu

† uli@alum.mit.edu

$\ddagger$ Authors are listed alphabetically - both authors are equally first authors. 
investigate typologically, to ask which is the actual state. This number is so large that the answer to the question which of these subsets can be derived from how many valid feature inventories cannot be visualized in the same way as in Table 3. To be able to still compare the three and the four cell results, we report therefore comparable summary figures for both the three cell case in table A1 and the four cell case in Table A2. We report here only the total count of different ordered $(\mathrm{O})$ and Pāninian $(\mathrm{P})$ partition sets using the abbreviations $O P S$ and $P P S$ : we define the $\operatorname{OPS}(I)$ for an inventory of features $I$ as the set of partitions such that the valid, ordered feature sequences drawn from the intersective closure of $F$ can generate all and only the partitions in OPS $(I)$. Similarly, the $\operatorname{PPS}(I)$ is the set of partitions such that the valid, Pāninian feature sequences drawn from the intersective closure of $F$ can generate all and only the partitions in $\operatorname{PPS}(I)$.

In the three cell case, the values shown in table A1 can be read off from table 3 except for the first two lines and the second column. The first column shows the size of the sets of features under consideration, ranging from zero to the size of the set of all features, i.e. $2^{c}-1$ where $c$ is the number of cells. The second column shows the number of subsets of that size whether valid or not, i.e. the binomial coefficient $\left(\begin{array}{c}2^{c}-1 \\ \# \text { features }\end{array}\right)$. The next column lists how many of these features sets are valid inventories. This corresponds to the sum over the values of the corresponding row in Table 3 - recall that valid feature sets are valid regardless of whether order is extrinsic or intrinsic. The penultimate column indicates how many different OPSs can be derived from the valid inventories. This corresponds to the number of non-zero entries in the corresponding row of Table 3. The last column shows the number of PPSs derivable from the valid inventories. The last row of Table 3 gives the values for feature sets of any size. The number of inventories and that of valid inventories in the last row are the sum of the entries for feature sets of a specific cardinality because there can't be any overlap. But the OPSs and PPSs can overlap - a paradigm set that is derivable from a three feature inventory can in some cases also be derived from a four feature inventory. Therefore the values in the last row of the \#PPS and \#OPS columns aren't the column sums.

For the four-cell paradigm space, the picture changes drastically. This is largely because describing a four cell space requires at least three features (as we show below). The table corresponding to Table A1 for four cells is shown as Table A2. We computed the table using an Apple Macbook Pro Laptop computer (2012 model) running a program in the $\mathrm{R}$ programming language ( $\mathrm{R}$ Core Team 2012) that tests all the feature sets for validity (computer code available, Sauerland 2017). The total computation time amounted to several hours. 


\begin{tabular}{crrrr} 
\#features & \#Inventories & \#validInventories & \#OPS & \#PPS \\
\hline 0 & 1 & 0 & - & - \\
1 & 7 & 0 & - & - \\
2 & 21 & 3 & 3 & 1 \\
3 & 35 & 29 & 12 & 9 \\
4 & 35 & 35 & 11 & 11 \\
5 & 21 & 21 & 5 & 5 \\
6 & 7 & 7 & 2 & 2 \\
7 & 1 & 1 & 1 & 1 \\
\hline any & 128 & 96 & 12 & 12 \\
\hline
\end{tabular}

Table A1 Summary statistics of logically possibly three cell partitions

\begin{tabular}{crrrr}
\hline \#features & \#Inventories & \#validInventories & \#OPS & \#PPS \\
\hline 0 & 1 & 0 & - & - \\
1 & 15 & 0 & - & - \\
2 & 105 & 0 & - & - \\
3 & 455 & 140 & 116 & 47 \\
4 & 1365 & 1015 & 317 & 239 \\
5 & 3003 & 2793 & 347 & 402 \\
6 & 5005 & 4935 & 310 & 420 \\
7 & 6435 & 6425 & 240 & 369 \\
8 & 6435 & 6435 & 160 & 279 \\
9 & 5005 & 5005 & 99 & 193 \\
10 & 3003 & 3003 & 54 & 112 \\
11 & 1365 & 1365 & 25 & 61 \\
12 & 455 & 455 & 12 & 24 \\
13 & 105 & 105 & 6 & 6 \\
14 & 15 & 15 & 2 & 2 \\
15 & 1 & 1 & 1 & 1 \\
\hline any & 32768 & 31692 & 361 & 463 \\
\hline
\end{tabular}

Table A2 Summary statistics of logically possibly four cell partitions 
The results for the four-cell systems differ noticeably from the three-cell case in terms of the effect of the Pānini assumption and the overall restrictiveness. The biggest difference is that in the three cell case the minimal inventories contain 2 features, but for four cells a least 3 features are required. Validity and Minimality are about equally restrictive, but in relation to the 256-times greater set of feature sets. This leaves 140 minimal valid feature inventories. Unlike the three-cell space, where adding Pānini (assuming closure under intersection) eliminated the possibility for variation, in the four-cell space, Pāṇinian sequences allow 47 distinct possible partition sets, each of which generates between 1 and 7 of the $B_{4}=15$ distinct partitions of a 4-celled space. None generate more than 7 partitions. The occurrence of the number 47 - a prime number that is not a Mersenne prime - in the table is, we think, noteworthy: ${ }^{1}$ It might indicate that even though a Pāninian sequence is a straightforward mathematical concept, the concept leads to some irreducible formal complexity. ${ }^{2}$ We include a display of these 47 paradigm sets as Appendix B.

A second main difference concerns the relation between the order-sensitive partition sets (OPSs) and Pạninian partition sets (PPSs). In the three cell case, the OPS (top half of Table 3) and PPS (bottom half of the table) were overall the same. But with four cells, sequences with extrinsic order and Pāninian sequences predict different restrictions on the paradigm sets. As shown by the last line of Table A2, the Pānini assumption allows slightly more variation - 463 paradigm sets are generable with Pāninian sequences, while only 361 are compatible with extrinsically ordered systems. ${ }^{3}$ We furthermore computed the amount of overlap between the OPSs and PPSs. We found that the union of OPSs and PPS contains 557 partition sets, and therefore 267 are both OPS and PPS, 94 are only OPSs, and 196 are only PPSs. Some examples are shown in Table A3: the first three rows show three of the 267 partition sets that are both OPSs and PPSs. The second block of three rows shows

1 We admit some bias here as at the time of writing this both authors were 47 years old. 2 Work in progress of ours in collaboration with Tom Green lends support this conclusion.

3 It may seem counter-intuitive that systems that allow any order of rules generate less variation than systems which are limited only to Pāninian rule orders. In terms of individual partitions, since Pāninian orders are a (proper) subset of the orders that are statable as extrinsic orders, Pāninian systems will not generate fewer partitions than those with unrestricted ordering. But what we count here is not the partitions that are generated, but the partition sets. In general, allowing extrinsic order will mean that the partition set generable from a given inventory will be a superset of the set generable from the same inventory with only Pāninian order. The numbers above reflect the effect that there is more convergence among the larger sets: with unrestricted rule ordering, more distinct inventories converge on the same partition sets than in the case of restricted rule ordering, overall. 


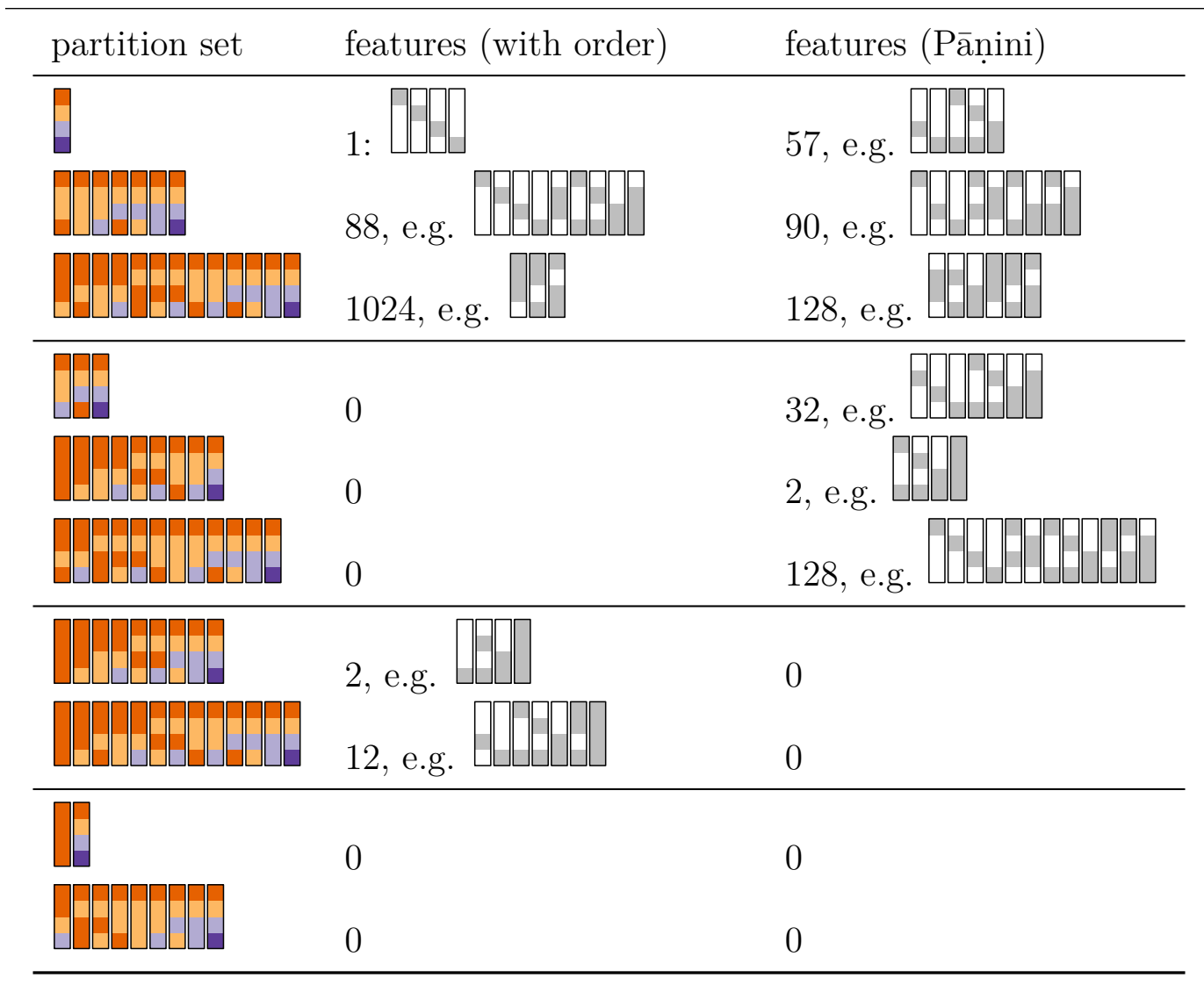

Table A3 Examples of four-cell partition sets with the number of ordered and Panini analyses and examples of feature inventories (assuming intersective closure)

three that are only PPSs, and the third block shows two that are only OPSs. The last block illustrates with two examples the 15827 partition sets that cannot be generated by a feature-based morphological system unless additional restrictions are introduced.

Overall, feature-based analyses are surprisingly restrictive in the four-cell case. Of the 16384 logically possible subsets of paradigms, only fewer than $3.4 \%$ (557: 16384) are compatible with a feature-based approach, 15827 logically conceivable typological states are ruled out. ${ }^{4}$ Pānini's assumption is even

4 One reviewer points out that permutations among the cells of a paradigm lead to multiple counting of equivalent patterns as was shown by the column grouping in Table 3 above. Since taking equivalence under permutation into account would reduce both the number of logical possibilities as well as that of OPSs and PPSs, we expect that it would not affect the 
slightly more restrictive, allowing fewer than $2.9 \%$ (463: 16384) of all possible sets of partitions. This is surprising since the feature assumption and also the Pānini assumption only ruled out 4 of 16 paradigm sets in the 3 cell case, i.e. $75 \%$ of the paradigm sets are compatible. Even assuming minimal Pāninisystems turns out to be more restrictive in the four cell than in the three cell case, allowing fewer than $0.3 \%(47: 16384)$ of the paradigm sets with four cells compared to 1 of 16 with three cells. The numerical comparisons need to be taken into account when evaluating feature-based morphological theories against typological data since the more restrictive a constraint is the less likely it is that is satisfied by chance.

In many ways, the constraints imposed by feature inventories on universal grammar are at this point difficult to grasp concretely, though future formal work may find a way to do so. We explore therefore a four cell version of the *ABA constraint, *ABBA (see Caha 2009; Smith et al. 2016 for empiricallybased proposals that exclude this pattern). There are two other permutations of the ABBA partition, $\mathrm{ABAB}$ and $\mathrm{AABB}$. We investigated therefore which partition sets containing all three of $\mathrm{ABBA}, \mathrm{ABAB}$ and $\mathrm{AABB}$, as well as $\mathrm{ABCD}$, can be generated from ordered and Pạnini inventories. There are $2^{11}$, i.e. 2048 such partition sets, but only 41 of them can be generated by feature inventories. Of those, 21 can be generated with either ordered or Pāninian systems, and a further 10 each with either only an ordered or only a Pāninian system. The examples in the third and fifth row of Table A3 illustrates two such systems. The system in row three predicts 13 partitions and can be generated from only three features if order is allowed. But if Pānini's restriction is obeyed, at least six features are necessary to generate this system. Row 5 shows an ABBA-system with the smallest number of features required to be generated in a Pāninian way, namely 4. So in the Pāṇini case, ABBA-systems require more than the minimal number of features as we also found for ABAsystems.

To conclude this section, consider briefly the seven cell paradigm. Because both 3 and 7 have $2^{n}-1$ cells for some $n$, the structures of the two paradigms are similar. The minimal number of features of a valid inventory for a seven cell paradigms is three. Any set of three features $f_{a}, f_{b}$, and $f_{c}$ where each basic feature contains 4 cells, each conjunction of two features contains 2 , and the conjunction of all three features contains 1 cell is valid. There are $7 !=5040$ such systems, and all have the same structure except for permutations of the cells. One system is shown in (1) along with its order-sensitive partition set

overall picture very much. Since the permutation-dependent calculations that we report are easier to verify, we decided to present those here. 
(OPS) - like in the 3-celled paradigm space, only the maximally differentiated paradigm is possible with Pạnini sequences. It is noticeable that of the 61 generable partitions in the OPS none contains only A and B - only paradigms with at least a three-way partition are possible. Since all OPSs are identical except for cell-permutations, we can derive in this way a general *AB constraint for seven cell paradigm spaces. I.e., if there are 7-celled spaces, then they cannot be limited only to bipartitions.

(1)
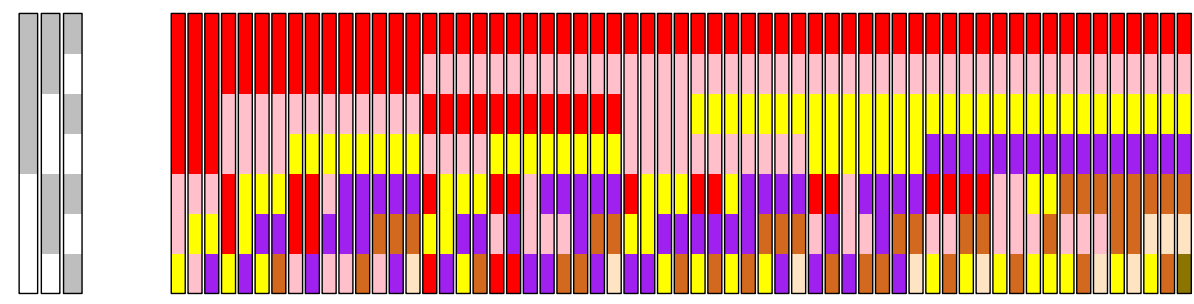


\section{B Appendix: The 47 Four Cell Pāninian Partition Sets (PPSs)}

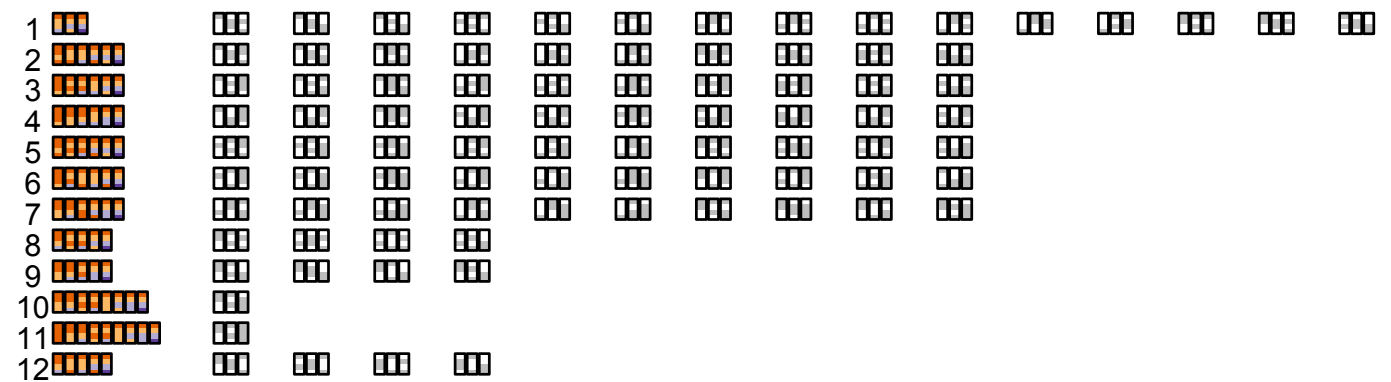

而

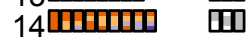

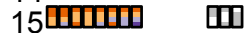

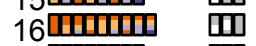

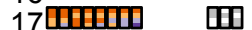

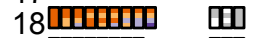

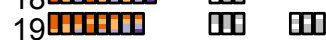

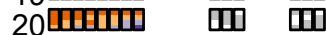

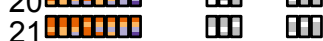

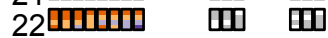

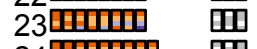

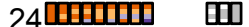

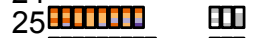

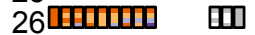

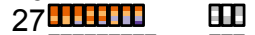

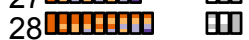

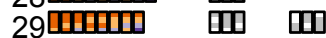

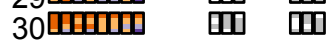

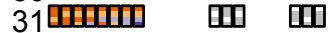

32क्षात्या एक

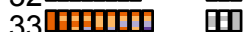

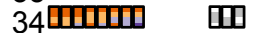

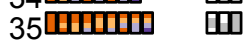

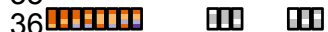

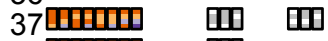

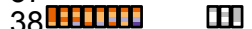

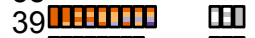

40प्ता एका एक

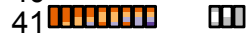

42प्सका एका एा

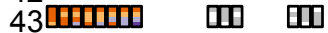

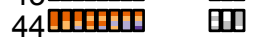

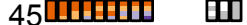

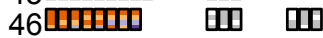

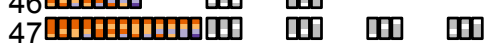

\section{References}

Caha, Pavel. 2009. The nanosyntax of case. Ph.D. thesis, CASTL Troms $\varnothing$. R Core Team. 2012. R: A language and environment for statistical computing. Vienna, Austria: R Foundation for Statistical Computing. 
Sauerland, Uli. 2017. Syncretism distribution modeling: R-code. URL http: //github.com/ulisauerland/sdm.

Smith, Peter, Beata Moskal, Ting Xu, Jungmin Kang \& Jonathan David Bobaljik. 2016. Case and number suppletion in pronouns. Manuscript Goethe Universität Frankfurt, University of Connecticut, and Syracuse University. 